\title{
ON GROUPOIDS DEFINED BY COMMUTATORS
}

\author{
KI HANG KIM AND FRED W. ROUSH ${ }^{1}$
}

\begin{abstract}
We study matrices $R, L$ which count the numbers of solutions of $i x=j$ and $x i=j$. For slight generalizations of $R, L$, the relation $R L=L R$ characterizes associativity of a groupoid. For groupoids defined by group commutators $x y x^{-1} y^{-1}$ the relation $R L=L R$ is valid. In addition one can study analogues of Green's relations. Any $g$-class contains at most four $\mathcal{H}$-classes in a commutator groupoid.
\end{abstract}

In this paper we mainly consider groupoids whose underlying set is a group, with groupoid multiplication $x * y=x y x^{-1} y^{-1}$. Our interest is mainly in the matrices $R$ and $L$ such that $r_{i j}$ counts the number of solutions of $i * x=j$ and $l_{i j}$ counts the number of solutions of $x * i=j$.

Definition. Let $G$ be a groupoid. Let $t, u$ be functions from $G$ to a commutative semiring $K$ with 0 . Then $R(t)$ is the matrix $\left(r_{i j}\right)$ for $i, j \in G$ such that $r_{i j}=\Sigma t(x)$, the summation being over all $x$ such that $i x=j$, if this sum is defined. And $L(u)$ is the matrix $\left(l_{i j}\right)$ such that $l_{i j}=\sum u(x)$, the summation being over all $x$ such that $x i=j$ if this sum is defined. Summations over the empty set are considered to be 0 . And we assume $0+k=k$ and $0 k=0$ for all $k \in K$.

In this paper we consider the two cases: (i) $G$ finite, $K=\mathbf{Z}^{+} \cup\{0\}$; (ii) $G$ arbitrary, $K$ the Boolean algebra $\{0,1\}$. The following proposition is essentially due to M. S. Putcha [2].

Proposition 1. In the two cases just mentioned, the matrices $R(t), L(u)$ commute for all $t, u$ if and only if $G$ is associative.

Proof. We have

$$
(R(t) L(u))_{i j}=\sum t(x) u(y)
$$

where the summation is over all pairs such that $i x=k, y k=j$ for some $k$, i.e. all pairs such that $y(i x)=j$. Likewise

$$
(L(u) R(t))_{i j}=\sum u(y) t(x)
$$

where the summation is over all pairs such that $(y i) x=j$. So if $G$ is

\footnotetext{
Received by the editors August 5, 1977.

AMS (MOS) subject classifications (1970). Primary $20 \mathrm{~L} 10$.

Key words and phrases. Brandt groupoid, commutator groupoid, Green's relation, Lie algebra, nilpotent, strongly connected graph.

${ }^{1}$ This research was supported by Alabama State University Grant R-78-6.
} 
associative $R(t), L(u)$ commute. For the converse, let $u, t$ range independently over all functions which send every element of $G$ except one, to zero. This proves the proposition.

REMARK. By choosing $t, u$ to send elements of $G$ to randomly chosen real numbers, this might give a quick computer test for nonassociativity of a groupoid.

From here on, we assume both $t, u$ send all elements of $G$ to 1 , and we write $R, L$ for $R(t), L(u)$.

Definition. A group commutator groupoid is a groupoid $G$ whose underlying set is a group and whose groupoid product is given by $x y x^{-1} y^{-1}$.

Proposition 2. Let $G$ be a group commutator groupoid. Let $T$ be the matrix of the permutation $x \rightarrow x^{-1}$. Then $R T=T R=L$. Therefore $R, L$ commute.

Proof. The equation $R T=L$ follows from $\left(i x i^{-1} x^{-1}\right)^{-1}=x i x^{-1} i^{-1}$. The identity $i^{-1} x i x^{-1}=\left(i^{-1} x i\right) i\left(i^{-1} x i\right)^{-1} i^{-1}$ implies $T R=R T$.

Proposition 3. For each $a, b, R_{a b}$ and $L_{a b}$ are each either zero or the order of the centralizer of $a$. The row sums of $R, L$ all equal the order of $G$. The bth column sum of $R$ and the bth column sum of $L$ each equal the number of pairs $x$, $y$ such that $x y x^{-1} y^{-1}=b$. The trace of $R$ equals the order of $G$. The trace of $L$ equals the sums of the orders of the centralizers of those elements a which are conjugate to $a^{2}$.

Proof. The entry $R_{a b}$ is the number of solutions of $x a^{-1} x^{-1}=a^{-1} b$. This is either zero or has the same order as the centralizer of $a^{-1}$. But the centralizer of $a$ equals the centralizer of $a^{-1}$. Likewise for $L_{a b}$. The second and third statements can be observed to be true. For the fourth statement, note that the trace of $R$ is the sum of the orders of the centralizers of such that $x a^{-1} x^{-1}=e$. But this can happen only if $a=e$. Likewise for $L$. This proves the proposition.

Definition. A (left, right) ideal in a groupoid is a subset closed under (left, right) multiplication. The principal (left, right) ideal generated by an element is the intersection of all (left, right) ideals containing that element. Two elements are $(\Re, \mathcal{L}, \mathcal{F}$ )-equivalent if and only if they generate the same principal (right, left, two-sided) ideal. They are $\mathcal{H}$-equivalent if and only if they are both $\Re$ - and $\mathcal{L}$-equivalent. These equivalence relations are called Green's relations.

Definition. A directed graph is strongly connected if and only if every point can be reached from every other point by a directed path.

Corresponding to this one can express any graph as a disjoint union of its strong components. We consider the graph of a matrix to be the graph whose vertices are the elements of the index set of the matrix, having an edge from $i$ to $j$ if and only if the $(i, j)$-entry of the matrix is nonzero.

Proposition 4. For any groupoid, the strong components of the graphs of 
$I+R, I+L,(I+R)(I+L)$ are the $R, \mathcal{L}, \mathcal{G}$-classes. Here $I$ denotes the identity matrix.

Note that if the elements of $G$ are arranged in the order of an ascending chain of normal subgroups, the matrices $R, L$ will assume a block triangular form. In addition nilpotency can easily be detected.

THEOREM 5. A finite group $G$ is nilpotent if and only if the matrix $R$ of its commutator groupoid is nilpotent. Likewise for $L$.

Proof. Suppose $G$ is nilpotent. Arrange the elements of $G$ in the order of an ascending central series. Then $R, L$ are lower subtriangular matrices.

Suppose $G$ is not nilpotent. Then by Theorem 14.4.7 of [1] there exist $x, p$ such that $x$ has order prime to $p$ and $x$ normalizes but does not centralize some $p$ subgroup $Q$. Choose $Q$ to be minimal. Then $x$ acts trivially on $[Q, Q]$ by conjugation. Then $x$ does not act trivially on $Q /[Q, Q]$ by conjugation, or the group generated by $x, Q$ would have a central series. So $x$ gives a nontrivial automorphism of $Q /[Q, Q]$. An endomorphism of $Q /[Q, Q]$ is given by $y \rightarrow x y x^{-1} y^{-1}, \bmod [Q, Q]$. If this endomorphism were nilpotent, the automorphism $x y x^{-1}$ would have order a power of $p$, which is false. Thus the endomorphism of $Q /[Q, Q]$ given by $y \rightarrow x y x^{-1} y^{-1}$ is not nilpotent. This implies $L$ is nonnilpotent. Similarly for $R$.

THEOREM 6. If $G$ is a group commutator groupoid, every $g$-class of $G$ contains at most two $\Re$-classes and at most two $\mathfrak{L}$-classes. If there are two of either type, they are equal in size. And $a g b$ if and only if there exists $c$ such

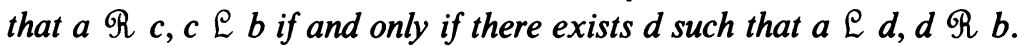

Proof. The classes will not be affected if we use matrices over the Boolean algebra $\{0,1\}$ always, The classes obtained from $I+R, I+L,(I+R)(I+$ $L)$ are the same as those obtained from

$$
\begin{aligned}
\bar{R} & =I+R+R^{2}+\ldots, \\
\bar{L} & =I+L+L^{2}+\ldots, \\
\bar{R} \bar{L} & =\sum_{0}^{\infty} R^{n}+\sum_{1}^{\infty} R^{n} T .
\end{aligned}
$$

Suppose $a g b$. Note that $\bar{R}, \bar{L}, \bar{R} \bar{L}$ are idempotent. Thus there is an edge in the graph of $\bar{R} \bar{L}$ from $a$ to $b$ and one from $b$ to $a$. Each of these two edges comes from one of the two summands

$$
\sum_{0}^{\infty} R^{n}, \quad \sum_{1}^{\infty} R^{n} T
$$

In the first case there is an $\bar{R}$ edge from one to the other and in the second case there is an $\bar{R}$ edge from one to the inverse of the other. We denote the existence of an edge from one to the other by $\rightarrow$. We observe that $x \rightarrow y^{-1}$ if and only if $x^{-1} \rightarrow y$ since $R T=T R$. There are four cases:

Case 1. $a \rightarrow b, b \rightarrow a$ in the graph of $\bar{R}$. Then $a \Re b$. 
Case 2. $a \rightarrow b^{-1}, b \rightarrow a^{-1}$ in the graph of $\bar{R}$. Then $a \Re b^{-1}$.

Case 3. $a \rightarrow b, b \rightarrow a^{-1}$ in the graph of $\bar{R}$. Then also $a^{-1} \rightarrow b^{-1}, b^{-1} \rightarrow a$. These imply $a \Re b$.

Case 4. $a \rightarrow b^{-1}, b \rightarrow a$. Again $a \Re b$. Therefore either $a$ lies in the $\Re$-class of $b$ or that of $b^{-1}$. Thus the $q$-class of $b$ contains at most two $\Re$-classes. Likewise it contains at most two $\mathcal{L}$-classes.

Suppose there do exist two $R$-classes in some $q$-class. Then there exist $a, b$ such that $a g b$ but not $a \Re b$. Thus the situation must be that of Case 2 . And for any $a, b$ in different $R$-classes but in the same $\mathcal{G}$-class, this must be so. Therefore $a \Re b^{-1}$. Thus for any $b$ in this $q$-class, $b$ and $b^{-1}$ will lie in different $\Re$-classes. Therefore the mapping $x \rightarrow x^{-1}$ will be a $1-1$ onto mapping from one $\Re$-class to the other. Likewise for $\mathcal{L}$-classes.

In Cases $1,3,4, a \Re b$ and the last statement is valid. Suppose we are in the second case. Suppose $a \rightarrow b^{-1}$ by an odd number of edges in the graph of

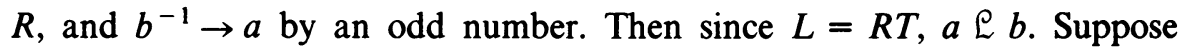
$a \rightarrow b^{-1}$ by an even number of $\Re$ edges and $b^{-1} \rightarrow a$ by an even number. Let $a \rightarrow x$ be the first edge in the sequence from $a$ to $b^{-1}$. Then $a \rightarrow x \rightarrow b^{-1}$ $\rightarrow a \rightarrow x$. So $a \Re x, x £ b$. And $a \varrho x^{-1}, x^{-1} \Re b$. If the number of edges from $a$ to $b^{-1}$ is even and the number of edges from $b^{-1}$ to $a$ is odd, or vice versa, we can double the path and obtain one of the two former cases. This proves the theorem.

EXAMPLE 1. For the symmetric group on three symbols, $L$ and $R$ are, respectively

$$
\left[\begin{array}{llllll}
6 & 0 & 0 & 0 & 0 & 0 \\
3 & 3 & 0 & 0 & 0 & 0 \\
3 & 0 & 3 & 0 & 0 & 0 \\
2 & 2 & 2 & 0 & 0 & 0 \\
2 & 2 & 2 & 0 & 0 & 0 \\
2 & 2 & 2 & 0 & 0 & 0
\end{array}\right],\left[\begin{array}{llllll}
6 & 0 & 0 & 0 & 0 & 0 \\
3 & 0 & 3 & 0 & 0 & 0 \\
3 & 3 & 0 & 0 & 0 & 0 \\
2 & 2 & 2 & 0 & 0 & 0 \\
2 & 2 & 2 & 0 & 0 & 0 \\
2 & 2 & 2 & 0 & 0 & 0
\end{array}\right]
$$

EXAMPLE 2. It is difficult to find a finite group with a $\mathcal{G}$-class containing four different $\mathcal{H}$-classes. Consider the semidirect product of the multiplicative group of numbers of the form $\pi^{i}(\pi-1)^{j}$ with the additive real numbers. Then $1 \mathcal{L} \pi-1$ but 1 and $\pi-1$ are not $\Re$-equivalent. Also $1 \Re 1-\pi$ but

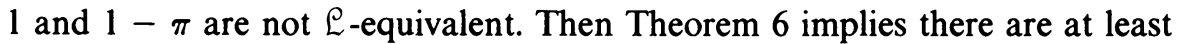
four distinct $\mathcal{H}$-classes, in the $\mathcal{G}$-class of 1 .

REMARK. Many of the results demonstrated here are trivially true for groupoids defined by Lie algebra commutators.

\section{REFERENCES}

1. Marshall Hall, The theory of groups, Macmillan, New York, 1959.

2. M. S. Putcha, Letter, Feb. 14, 1977.

Department of Mathematics, Alabama State University, Montgomery, Alabama 36101 